\title{
Neuroimaging basis in the conversion of aMCl patients with APOE- $\varepsilon 4$ to AD: study protocol of a prospective diagnostic trial
}

\author{
Guan-Qun Chen', Can Sheng', Yu-Xia Li ${ }^{1,3}$, Yang Yu', Xiao-Ni Wang ${ }^{1}$, Yu Sun ${ }^{1}$, Hong-Yan Li ${ }^{1,4}$, Xuan-Yu Li ${ }^{1}$, \\ Yun-Yan Xie ${ }^{1}$ and Ying $\operatorname{Han}^{1,2^{*}}$
}

\begin{abstract}
Background: The $\varepsilon 4$ allele of the Apolipoprotein E gene (APOE- $\varepsilon 4)$ is a potent genetic risk factor for sporadic Alzheimer's disease (AD). Amnestic mild cognitive impairment (aMCl) is an intermediate state between normal cognitive aging and dementia, which is easy to convert to AD dementia. It is an urgent problem in the field of cognitive neuroscience to reveal the conversion of aMCl- $\varepsilon 4$ to $A D$. Based on our preliminary work, we will study the neuroimaging features in the special group of $\mathrm{aMCl}-\varepsilon 4$ with multi-modality magnetic resonance imaging (structural $\mathrm{MRI}$, resting state-fMRI and diffusion tensor imaging) longitudinally.

Methods/Design: In this study, 200 right-handed subjects who are diagnosed as aMCI with APOE- $\varepsilon 4$ will be recruited at the memory clinic of the Neurology Department, XuanWu Hospital, Capital Medical University, Beijing, China. All subjects will undergo the neuroimaging and neuropsychological evaluation at a 1 year-interval for 3 years. The primary outcome measures are 1) Microstructural alterations revealed with multimodal MRI scans including structure MRI (sMRI), resting state functional MRI (rs-fMRI), diffusion tensor imaging (DTI); 2) neuropsychological evaluation, including the World Health Organization-University of California-LosAngeles Auditory Verbal Learning Test (WHO-UCLA AVLT), Addenbrook's cognitive examination-revised (ACE-R), mini-mental state examination (MMSE), Montreal Cognitive Assessment (MoCA), Clinical Dementia Rating scale (CDR).
\end{abstract}

Discussion: This study is to find out the neuroimaging biomarker and the changing laws of the marker during the progress of $a M C l-\varepsilon 4$ to $A D$, and the final purpose is to provide scientific evidence for new prevention, diagnosis and treatment of $\mathrm{AD}$.

Trial Registration: This study has been registered to ClinicalTrials.gov (NCT02225964, https://www.clinicaltrials.gov/) in August 24, 2014.

Keywords: APOE4 allele, Alzheimer's disease, Amnestic mild cognitive impairment, Neuroimaging techniques, Multimodality, Longitudinal study

\section{Background}

Alzheimer's disease (AD) is a common neurodegenerative disorder, which is increasingly prevalent among the elderly. AD patients usually complain of memory impairment and serious decline of the daily living abilities. The aMCI is an intermediate state between normal cognitive aging

\footnotetext{
* Correspondence: hanying@xwh.ccmu.edu.cn

'Department of Neurology, XuanWu Hospital of Capital Medical University, Beijing 100053, China

${ }^{2}$ Center of Alzheimer's Disease, Beijing Institute for Brain Disorders, Beijing 100053, China

Full list of author information is available at the end of the article
}

and dementia, which is easily progressed to AD dementia. Thus, the effective therapy of AD is to be delivered once the diagnosis is made at the very early stages of AD, ideally in the phase of aMCI. The human apolipoprotein $\mathrm{E}$ (APOE) gene has three polymorphic alleles (E2, E3, and E4) [1]. APOE- $\varepsilon 4$ was confirmed to be related with sporadic $\mathrm{AD}$ risks by increasing the risk of developing $\mathrm{AD}$ and reducing the age of onset [2]. Therefore, it is an urgent requirement in the field of cognitive neuroscience to reveal the underlying neurological mechanism of the conversion of aMCI to AD in the patients with APOE- $\varepsilon 4$. 
Although the cognition evaluation is simple, the accuracy of the diagnosis depends on the professional skills of the doctor and the collaboration of patients. On the other hand, MRI might be a promising strategy for early diagnosis of AD [3]. MRI might be the best diagnosis method for AD by mapping both structures and functions of the human brain. Structurally, studies showed that brain atrophy in $\mathrm{AD}$ patients first appears in the medial temporal lobe (MTL), and then involves the parietal lobe, frontal lobe and occipital lobe, and finally the anterior cingulate cortex $[4,5]$. Consistent with these findings, evidence from our team has demonstrated the progressive alterations of brain structures during the progression to AD [6]. Compared with APOE- $\varepsilon 4$ non-carriers, the carriers exhibited greater medial temporal lobe atrophy [7]. APOE- $\varepsilon 4$ is an independent risk factor for the hypotrophy hippocampus in patients with AD and MCI [8]. Decreased gray matter volume mainly restricts in the right brain area for aMCI- $\varepsilon 4$ patients [9]. Not the degree of atrophy, but the rate of atrophy in a certain region of brain demonstrate close relationship with the AD progression [10]. The rate of hippocampal atrophy is suggested to be an early marker of incipient memory decline and dementia [11]. The longitudinal study indicated that rate of volumetric loss was significantly greater among MCI- $\varepsilon 4$ compared with non-carriers [12]. Compared with structural MRI, diffusion tensor imaging (DTI) is a more sensitive method for detecting white matter damage [13]. A shortterm follow-up study showed that AD patients had lower fractional anisotropy (FA) than controls in the fornix and anterior portion of the cingulum bundle, and these FA values were positively correlated with cognitive score [14]. The APOE- $\varepsilon 4$ modulates white matter (WM) before clinical manifestations and cognitive impairment [15]. The changes of white matter is accompanied with the cognitive function decline in APOE- $\varepsilon 4$ carriers [16]. Previous studies suggested that rs-fMRI is a potential functional biomarker for cognitive impairment. The aMCI subjects showed that posterior cingulate cortex/precuneus (PCC/ $\mathrm{PCu}$ ) hyper-functional connectivity was found at baseline, while a substantial decrease of these connections was observed at follow-up [17]. Our team observed that the aMCI patients had decreased amplitude of low-frequency fluctuations (ALFF) values in the $\mathrm{PCC} / \mathrm{PCu}$, anterior medial prefrontal cortex (aMPFC), hippocampus/parahippocampal gyrus (PHG), basal ganglia, and prefrontal regions, and increased ALFF values mainly in several occipital and temporal regions [18]. APOE-ع4 exerts an influence on brain function in patients with MCI. Using whole-brain pulsed arterial spin labeling (ASL) magnetic resonance imaging suggest that cognitive status and APOE genotype have interactive effects on cerebral blood flow (CBF) [19]. MCI-ع4 patients demonstrated significantly increased CBF [20]. However, deficits exist in these studies that using rs-fMRI to reveal the effect of APOE- 44 on MCI patients.

For the purpose of early diagnosis, the researchers have been focusing on the aMCI window. Although previous studies provide important information for the conversion of aMCI to $\mathrm{AD}$, they were based on the single modality neuroimaging analysis. Moreover, previous studies ignored one of the major factors that affects the course of the MCI converting to AD is the APOE- $\varepsilon 4$. Thus, the purpose of the current trial is to longitudinally investigate the multi-modal (structural MRI, resting state-fMRI and diffusion tensor imaging) neuroimaging characteristics of aMCI- $\varepsilon 4$ patients. We hope to provide scientific evidence for more effective prevention, diagnosis and treatment of AD.

\section{Methods/Design}

The scheme of the current prospective trial is described in Fig. 1.

\section{Subject}

1. Subjects inclusion: subjects with aMCI included the following: (1) memory complaint, preferably confirmed by an informant; (2) a single domain or multi domain cognitive decline; abnormal objective memory impairment documented by the scores falling 1.5 SD below the age and education matched-specific norms on memory test; being free from dementia according to the Diagnostic and Statistical Manual of Mental Disorders, Fourth Edition, revised (DSM-IV-R); (3) objective memory impairment, cutoff points of MiniMental State Examination (MMSE) score: 19 (no formal education), 22 (1 to 6 years of education), and 26 (7 or more years of education); cutoff points of Montreal Cognitive Assessment (MoCA): 13 (no formal education), 19 (1 to 6 years of education), and 24 (7 or more years of education) [21]; a Clinical Dementia Rating (CDR) score of 0.5; (4) the Han nationality, right-handed (the Edinburgh handedness scale score $>40$ points); no fixed dentures and can accept MRI scan; (5) able to complete 3 years follow-up.

2. Subjects exclusion: (1) those who have a clear history of stroke; (2) severe depression that led to mild cognitive impairment (Hamilton Depression Rating Scale score $\geq 24$ points); (3) other nervous system diseases, which can cause cognitive impairment (such as brain tumors, Parkinson's disease, encephalitis, and epilepsy); (4) cognitive impairment caused by traumatic brain injury; (5) other systemic diseases, which can cause cognitive impairment, such as thyroid dysfunction, severe anemia, syphilis, and HIV; (6) a history of 


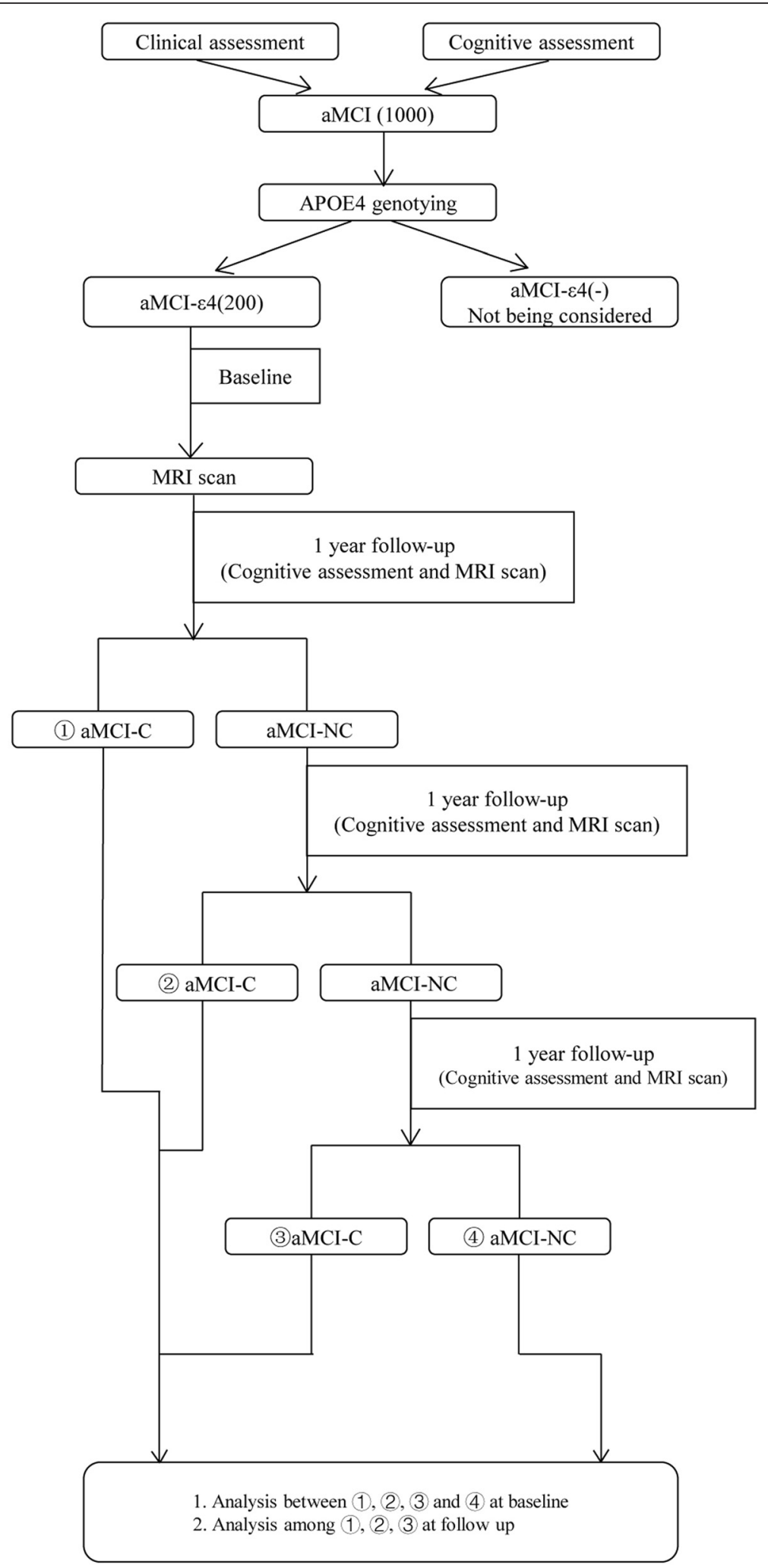

Fig 1 Flowchart of the current prospective diagnostic trial 
psychosis or congenital mental growth retardation; and (7) subjects with contraindications for MRI.

\section{Neuropsychological assessments}

All subjects will receive a standardized clinical and neuropsychological evaluation, including WHO-UCLA AVLT, ACE-R, MMSE, MoCA, CDR.

\section{APOE genotype}

After the neuropsychological assessments and before the MRI scan, genomic DNA will be extracted from peripheral blood using a Blood Genomic DNA Extraction Kit. The APOE genotypes will be determined by a restriction enzyme polymerase chain reaction technique. Only subjects carrying the gene of E3/E4 or E4/E4 will be enrolled in this study group.

\section{Magnetic resonance brain imaging Imaging protocol}

Imaging data will be acquired using a $3.0 \mathrm{~T}$ Trio Siemens scanner at XuanWu Hospital, Capital Medical University. For each participant, conventional brain T1-weighted (T1WI), T2-weighted (T2WI) and fluid-attenuated inversion recovery (FLAIR) images will be obtained to exclude serious brain diseases. Brain MR images will be inspected by an experienced neuroradiologist (with over 5-year experience).

sMRI. using a sagittal MP-RAGE sequence with the following imaging parameters: $\mathrm{TR}=1900 \mathrm{~ms}$; $\mathrm{TE}=2.2 \mathrm{~ms}$; inversion time $=900 \mathrm{~ms}$; flip angle $=9^{\circ}$; FOV $=256 \mathrm{~mm} \times$ $256 \mathrm{~mm}$; matrix $=256 \times 256 ; 176$ slices, thickness $=1.0 \mathrm{~mm}$.

rs-fMRI. using an echo-planar imaging sequence with the following parameters: repetition time $(\mathrm{TR})=2000 \mathrm{~ms}$, echo time $(\mathrm{TE})=40 \mathrm{~ms}$, flip angle $(\mathrm{FA})=90^{\circ}$, number of slices $=28$, slice thickness $=4 \mathrm{~mm}$, gap $=1 \mathrm{~mm}$, voxel size $=4 \times 4 \times 4 \mathrm{~mm}^{3}$, and matrix $=64 \times 64$. Participants will be asked to lie quietly in the scanner with their eyes closed during data acquisition. Each scan lasted for $478 \mathrm{~s}$.

DTI. using an echo planar imaging (EPI) sequence in 32 independent, non-collinear directions of a b-value = $1000 \mathrm{~s} / \mathrm{mm}^{2}$, and one additional image with no diffusion weighting $(\mathrm{b}=0)$. $\mathrm{TR}=11000 \mathrm{~ms}, \mathrm{TE}=98 \mathrm{~ms}$, flip angle $=90^{\circ}$, field of view $(\mathrm{FOV})=256 \mathrm{~mm} \times 256 \mathrm{~mm}$, imaging matrix $=128 \times 128$, number of slices $=60$, and slice thickness $=2 \mathrm{~mm}$. Three acquisitions will be averaged to increase the signal-to-noise ratio.

\section{MRI image analysis}

sMRI data analysis. Voxel-based morphometry (VBM) with DARTEL will be used to characterize gray matter volume (GMV). Voxel-based morphometry with DARTEL will be performed using SPM8 (Welcome Trust Center for Neuroimaging, London, UK, http://www.filion.ucl.ac.uk/ $\mathrm{spm} /$ software/spm8/). For the analysis of cortical thickness, we will use the software package of the Montreal Neurological Institute (MNI)(http://wiki.bic.mni.mcgill.ca/index.php/CIVET). This process includes structural image non-uniformity correction, spatial standardization, tissue segmentation (gray matter, white matter and cerebrospinal fluid), internal and external surface extraction of gray matter as well as the definition and measurement of cortical thickness.

rs-fMRI data analysis. Image preprocessing will be performed by using SPM8 (http://www.fil.ion.ucl.ac.uk/ $\mathrm{spm} /$ ) and Data Processing Assistant for Resting-State fMRI. The preprocessing procedures will be performed including removal of the first 10 volumes, slice timing, and head motion correction. All rs-fMRI data will be satisfied the criteria of spatial movement in any direction $<3 \mathrm{~mm}$ or $3^{\circ}$ and the subjects will be demonstrated no significant group differences in the head motion parameters (i.e, three translation and three rotation parameters). To normalize the fMRI data spatially, the T1-weighted images will be firstly registered to the mean functional data, and the resulting aligned T1 data set will be segmented and transformed into MNI space using the DARTEL toolbox, and a group template will be generated. Next, the motioncorrected functional volumes will be specially normalized to the group template using the transfer parameter estimated by DARTEL segmentation and resampled to $3 \mathrm{~mm}$ isotropic voxels. Further, the functional images will be spatially smoothed with a $4 \mathrm{~mm}$ Gaussian kernel. The linear detrend and temporal bandpass filtering (0.01 $0.08 \mathrm{~Hz}$ ) will be performed to reduce the influences of lowfrequency drift and high-frequency physiological noise. Finally, several nuisance signals will be regressed out from the data, including the six motion parameters, the global, the white matter, and the cerebrospinal fluid signals.

Functional connectivity (FC) analysis using DPARSF software (http://www.restfmri.net/forum/DPARSF).

DTI data analysis. DTI data processing will be carried out using FSL software (FMRIB Software Library, http:// www.fmrib.ox.ac.uk/fsl). Initially, eddy current correction will be run to correct gradient-coil distortions and small-head motions using affine registration to a reference image ( $\mathrm{b} 0$ volume). The brain voxels of DTI data will be extracted using the Brain Extraction Tool (BET). The maps of diffusion tensor parameters including FA and MD will be calculated using DTI-FIT tool, which fits a diffusion tensor model to diffusion-weighted images for each voxel. Voxel-wise statistical analysis of the FA and MD data will be performed for regional differences using TBSS.

\section{Follow up}

All aMCI- $\varepsilon 4$ patients will undergo a follow-up review of approximately 3 years with 1 -year interval. They will get the 
entire clinical examination, neuropsychological assessment and MRI scan during the baseline period. Individuals will come back to the XuanWu Hospital once 1 year for countercheck. All the evaluations are the same as that they receive during the first clinic visit. According to the diagnosis in the follow-up stage, aMCI- $\varepsilon 4$ patients will be divided into converters and nonconverters to $\mathrm{AD}$.

\section{Sample size calculation and statistics}

A total of 1000 right-handed aMCI patients will be recruited in this study. aMCI- $\varepsilon 4$ patients is about $26.6 \%$, and annual conversion rate of aMCI- $\varepsilon 4$ patients convert is $15.9 \%$ [22]. Thus, we plan to recruit 200 patients with aMCI- $\varepsilon 4$. There will be about 90 aMCI- $\varepsilon 4$ converted into AD after 3 years of follow-up.

Demographics and clinical characteristics of the subjects will be analyzed using SPSS 17.0. Two-sample two-tail $t$ test or two-tail Pearson chi-square test will be used to explore differences of these data among two groups (converters and nonconverters). $P<0.05$ is considered as with statistical significance.

For images analysis. To determine the difference between two groups (converters and nonconverters), we will perform a two-sample $t$-test. The significant level will be set at $P<0.05$.

To determine the difference between intra-groups, we will perform a One-way analysis of variance (ANOVA). Further, Dunnett- $t$ test will be performed for differences among intra-groups. The statistical threshold of the two test will both be set at $P<0.05$.

\section{Discussion}

To our know, APOE- $\varepsilon 4$ is a strong risk factor of AD. However, there has controversy about the effect of APOE- $\varepsilon 4$ on the conversion of aMCI- $\varepsilon 4$ to AD. A multivariate Cox regression model demonstrated that possession of an APOE- $\varepsilon 4$ was the strongest predictor of clinical progression among aMCI [23]. Other studies of have found limited clinical applicability for prediction of outcome among aMCI [24, 25]. Moreover, Stern et al implied that APOE- $\varepsilon 4$ is associated with a less aggressive form of AD [26]. Thus, previous studies have shown that simply depending on the psychometric tests will cause a noisy clinical data, and need biomarker to clarify the pathogenic mechanism of APOE- $\varepsilon 4$, such as MRI, PET (positron emission tomography), and cerebrospinal fluid (CSF) examinations. Comparing to PET and CSF examinations, MRI - a noninvasive, nonradiation means - has been widely used with easy patient acceptance in both clinical and scientific studies.

Some limitations have to be took into account about this study. First, the trial's follow up time may be too short to be sured whether MCI-nonconverter would convert to $\mathrm{AD}$ in the future. Therefore, we will plan to continue to track the dynamic situations of these patients. Second, there has many technical problems of MRI. Data acquisition, data processing and data analysis of MRI are lack of standards. So far, no MRI model can be used for clinical diagnosis of AD. But we deeply believe that with the development of neuroimaging, diagnosis of AD with MRI will achieve better performance.

Here, this present study is to find out the neuroimaging biomarker and the changing laws of the marker during the progress of aMCI- $\varepsilon 4$ to $\mathrm{AD}$, and the final purpose is to provide scientific evidence for new prevention, diagnosis and treatment of AD.

\section{Ethics approval and consent to participate}

Ethical approval of this has been obtained from the medical research ethics committee and institutional review board of XuanWu Hospital, Capital Medical University (approval number: [2014]011). All participation is based on written informed consent and the participants will be able to withdraw from the study at any time.

\section{Consent for publication}

Written consent is obtained from each subject before publishing in this study.

\section{Availability of data and material}

Not applicable.

\begin{abstract}
Abbreviations
AD: Alzheimer's disease; ALFF: amplitude of low-frequency fluctuations; aMCl: amnestic mild cognitive impairment; aMPFC: anterior medial prefrontal cortex; ANOVA: one-way analysis of variance; APOE- $\varepsilon 4$ : $\varepsilon 4$ allele of the Apolipoprotein E gene; ASL: arterial spin labeling; CDR: clinical dementia rating scale; CSF: cerebrospinal fluid; DPARSF: data processing assistant for resting-state $\mathrm{fMRl}$; DTI: diffusion tensor imaging; FA: fractional anisotropy; FC: functional connectivity; FLAIR: fluid-attenuated inversion recovery; MD: mean diffusivity; MMSE: mini-mental state examination; MoCA: Montreal cognitive assessment; MRI: magnetic resonance imaging; MTL: medial temporal lobe; PCC: posterior cingulate cortex; PCu: precuneus; PET: positron emission tomography; PHG: parahippocampal gyrus; rs-fMRI: resting state functional magnetic resonance imaging; sMRI: structural magnetic resonance imaging; SPM8: statistical parametric mapping 8; T1Wl: T1-weighted imaging; T2Wl: T2-weighted imaging; TBSS: tract-based spatial statistics; VBM: voxel-based morphometry; WHO-UCLA AVLT: World Health Organization- University of California-LosAngeles Auditory Verbal Learning Test; WM: white matter.
\end{abstract}

\section{Competing interests}

The authors declare that they have no competing interests.

\section{Authors' contributions}

All authors participated in the design of the study. CGQ drafted the manuscript. HY is supervising the project and made critical revision of the manuscript for important intellectual content. All authors read and approved the final manuscript.

\section{Acknowledgements}

The study was financially supported the Natural Science Foundation of China (No. 31371007 to Dr. Ying Han).

\section{Funding}

This study has been received financial support from the Natural Science Foundation of China (No. 31371007 to Dr. Ying Han) but not a commercial 
organization. The funding source have no role in the design and conduct of this study and the writing of this manuscript

\section{Author details}

'Department of Neurology, XuanWu Hospital of Capital Medical University, Beijing 100053, China. ${ }^{2}$ Center of Alzheimer's Disease, Beijing Institute for Brain Disorders, Beijing 100053, China. ${ }^{3}$ Department of Neurology, Tangshan Gongren Hospital, Tangshan 063000, China. ${ }^{4}$ Department of Neurology, Civil Aviation General Hospital, Beijing 100123, China.

\section{Received: 23 March 2016 Accepted: 5 May 2016}

\section{Published online: 12 May 2016}

\section{References}

1. Saunders AM, Strittmatter WJ, Schmechel D, George-Hyslop PH, PericakVance MA, Joo SH, Rosi BL, Gusella JF, Crapper-MacLachlan DR, Alberts MJ, et al. Association of apolipoprotein E allele epsilon 4 with late-onset familial and sporadic Alzheimer's disease. Neurology. 1993;43(8):1467-72.

2. Corder EH, Saunders AM, Strittmatter WJ, Schmechel DE, Gaskell PC, Small $G W$, Roses AD, Haines JL, Pericak-Vance MA. Gene dose of apolipoprotein E type 4 allele and the risk of Alzheimer's disease in late onset families. Science. 1993:261(5123):921-3.

3. Dubois B, Feldman HH, Jacova C, Dekosky ST, Barberger-Gateau P, Cummings J, Delacourte A, Galasko D, Gauthier S, Jicha G, et al. Research criteria for the diagnosis of Alzheimer's disease: revising the NINCDS-ADRDA criteria. Lancet Neurol. 2007:6(8):734-46.

4. Kaye JA, Swihart T, Howieson D, Dame A, Moore MM, Karnos T, Camicioli R, Ball M, Oken B, Sexton G. Volume loss of the hippocampus and temporal lobe in healthy elderly persons destined to develop dementia. Neurology. 1997:48(5):1297-304

5. McDonald CR, McEvoy LK, Gharapetian L, Fennema-Notestine C, Hagler Jr DJ, Holland D, Koyama A, Brewer JB, Dale AM. Regional rates of neocortical atrophy from normal aging to early Alzheimer disease. Neurology. 2009;73(6): 457-65.

6. Han Y, Lui S, Kuang W, Lang Q, Zou L, Jia J. Anatomical and functional deficits in patients with amnestic mild cognitive impairment. PLoS One. 2012:7(2):e28664.

7. Hua X, Leow AD, Parikshak N, Lee S, Chiang M, Toga AW, Jack CR, Weiner MW, Thompson PMTL. Tensor-based morphometry as a neuroimaging biomarker for Alzheimer"s disease: an MRI study of $676 \mathrm{AD}, \mathrm{MCl}$, and normal subjects. Neuroimage. 2008:43(3):458-69.

8. Andrawis JP, Hwang KS, Green AE, Kotlerman J, Elashoff D, Morra JH, Cummings JL, Toga AW, Thompson PM, Apostolova LG. Effects of ApoE4 and maternal history of dementia on hippocampal atrophy. Neurobiol Aging. 2012;33(5):856-66

9. Goni J, Cervantes S, Arrondo G, Lamet I, Pastor P, Pastor MA. Selective brain gray matter atrophy associated with APOE epsilon4 and MAPT H1 in subjects with mild cognitive impairment. J Alzheimers Dis. 2013;33(4):1009-19.

10. Scahill Rl, Schott JM, Stevens JM, Rossor MN, Fox NC. Mapping the evolution of regional atrophy in Alzheimer's disease: unbiased analysis of fluid-registered serial MRI. Proc Natl Acad Sci U S A. 2002;99(7):4703-7.

11. den Heijer T, van der Lijn F, Koudstaal PJ, Hofman A, van der Lugt A, Krestin GP, Niessen WJ, Breteler MM. A 10-year follow-up of hippocampal volume on magnetic resonance imaging in early dementia and cognitive decline. Brain. 2010;133(Pt 4):1163-72.

12. Moffat SD, Szekely CA, Zonderman AB, Kabani NJ, Resnick SM. Longitudinal change in hippocampal volume as a function of apolipoprotein E genotype. Neurology. 2000;55(1):134-6.

13. Charlton RA, Schiavone F, Barrick TR, Morris RG, Markus HS. Diffusion tensor imaging detects age related white matter change over a 2 year follow-up which is associated with working memory decline. J Neurol Neurosurg Psychiatry. 2010;81(1):13-9.

14. Mielke MM, Kozauer NA, Chan KC, George M, Toroney J, Zerrate M, Bandeen-Roche K, Wang MC, Vanzijl P, Pekar JJ et al. Regionally-specific diffusion tensor imaging in mild cognitive impairment and Alzheimer's disease. Neuroimage. 2009:46(1):47-55.

15. Heise V, Filippini N, Ebmeier KP, Mackay CE. The APOE varepsilon4 allele modulates brain white matter integrity in healthy adults. Mol Psychiatry. 2011;16(9):908-16.

16. Westlye ET, Hodneland E, Haasz J, Espeseth T, Lundervold A, Lundervold AJ. Episodic memory of APOE epsilon4 carriers is correlated with fractional anisotropy, but not cortical thickness, in the medial temporal lobe. Neuroimage. 2012;63(1):507-16.

17. Bai F, Watson DR, Shi Y, Wang Y, Yue C, YuhuanTeng, Wu D, Yuan Y, Zhang Z. Specifically progressive deficits of brain functional marker in amnestic type mild cognitive impairment. PLoS One. 2011;6(9), e24271.

18. Han Y, Wang J, Zhao Z, Min B, Lu J, Li K, He Y, Jia J. Frequency-dependent changes in the amplitude of low-frequency fluctuations in amnestic mild cognitive impairment: a resting-state fMRI study. Neuroimage. 2011;55(1): 287-95.

19. Wierenga CE, Dev SI, Shin DD, Clark LR, Bangen KJ, Jak AJ, Rissman RA, Liu TT, Salmon DP, Bondi MW. Effect of mild cognitive impairment and APOE genotype on resting cerebral blood flow and its association with cognition. J Cereb Blood Flow Metab. 2012;32(8):1589-99.

20. Bangen K, Restom K, Liu TT, Wierenga CE, Jak AJ, Salmon DP, Bondi MW. Assessment of Alzheimer's disease risk with functional magnetic resonance imaging: an arterial spin labeling study. J Alzheimers Dis. 2012;31 Suppl 3:S59-74.

21. Lu J, Li D, Li F, Zhou A, Wang F, Zuo X, Jia XF, Song H, Jia J. Montreal cognitive assessment in detecting cognitive impairment in Chinese elderly individuals: a population-based study. J Geriatr Psychiatry Neurol. 2011;24(4): 184-90.

22. Wang PN, Hong CJ, Lin KN, Liu HC, Chen WT. APOE- $\varepsilon 4$ increases the risk of progression from amnestic mild cognitive impairment to Alzheimer's disease among ethnic Chinese in Taiwan. Neurol Neurosurg Psychiatry. 2011:82(2):165-9.

23. Petersen RC, Smith GE, Ivnik RJ, Tangalos EG, Schaid DJ, Thibodeau SN, Kokmen E, Waring SC, Kurland LT. Apolipoprotein E status as a predictor of the development of Alzheimer's disease in memory-impaired individuals. JAMA. 1995:273(16):1274-8.

24. Devanand DP, Pelton GH, Zamora D, Liu X, Tabert MH, Goodkind M, Scarmeas N, Braun I, Stern Y, Mayeux R. Predictive utility of apolipoprotein E genotype for Alzheimer disease in outpatients with mild cognitive impairment. Arch Neurol. 2005;62(6):975-80

25. Barabash A, Marcos A, Ancin I, Vazquez-Alvarez B, de Ugarte C, Gil P, Fernandez C, Encinas M, Lopez-lbor JJ, Cabranes JA. APOE, ACT and CHRNA7 genes in the conversion from amnestic mild cognitive impairment to Alzheimer's disease. Neurobiol Aging. 2009;30(8):1254-64.

26. Stern Y, Brandt J, Albert M, Jacobs DM, Liu X, Bell K, Marder K, Sano M, Albert S, Del-Castillo CC, et al. The absence of an apolipoprotein epsilon4 allele is associated with a more aggressive form of Alzheimer's disease. Ann Neurol. 1997:41(5):615-20.

\section{Submit your next manuscript to BioMed Central and we will help you at every step:}

- We accept pre-submission inquiries

- Our selector tool helps you to find the most relevant journal

- We provide round the clock customer support

- Convenient online submission

- Thorough peer review

- Inclusion in PubMed and all major indexing services

- Maximum visibility for your research

Submit your manuscript at www.biomedcentral.com/submit
) Biomed Central 